\title{
Énergie marémotrice : Aspects liés à l'environnement aquatique
}

Tidal power : repercussions on the aquatic environment

\author{
C. Rétière
}

\author{
Muséum d'histoire naturelle \\ Directeur du laboratoire maritime de Dinard
}

P. Massonnet et F. Bordet

Département Sites-Environnement-Information Électricité de France

\author{
J.F. Parent
}

Région d'équipement Clamart Électricité de France

\section{Présentation générale \\ (P. Massonnet)}

Toutes les opérations relatives à la réalisation d'un grand projet tel, par exemple, qu'une usine marémotrice : choix du site, conception, construction et enfin exploitation, sont accompagnées d'études nombreuses concernant la protection de l'environnement.

Ces études sont aujourd'hui concrétisées aux travers des documents exigés par les diverses procédures administratives d'autorisation. Il s'agit essentiellement des " études d'impact " introduites par la loi sur la protection de la nature du 10 juillet 1976 , qui ont pour but d'évaluer dès la conception, l'impact du projet sur l'environnement et de s'assurer que celui-ci est acceptable.

Ces dispositions réglementaires favorisent une meilleure information du public, des élus locaux et associations et de l'Administration que par le passé, grâce à une connaissance plus approfondie des problèmes posés et des solutions qui peuvent être apportées pour les résoudre.

Electricité de France est particulièrement sensibilisé à ces problèmes d'environnement. Cette sensibilisation s'est traduite par la signature, le $1^{\text {er }}$ juillet 1982, d'une Convention entre l'Etablissement, le Ministère de l'Environnement et le Ministère de l'Industrie et de la Recherche, affirmant ainsi la volonté de l'Etablissement de faire un effort exemplaire en faveur de l'Environnement et de diriger cet effort vers des objectifs concrets répondant à l'intérêt général.

Il n'est pas inutile, je pense, de rappeler le montant des dépenses consenties par l'Etablissement pour l'environnement : 1400 MF en 1982.

Pour revenir à l'objet de cette journée consacrée à l'énergie marémotrice, et plus particulièrement aux aspects liés à l'environnement aquatique, je présenterai rapidement, les deux exposés qui vont suivre :

- les conséquences écologiques de la construction et $\mathrm{du}$ fonctionnement de l'usine de la Rance (Professeur C. Rétière); l'usine de la Rance est en service depuis plus de 15 ans. Quel est l'impact réellement constaté de cette réalisation sur l'environnement aquatique de la Rance ?

- les études qu'il convient d'engager pour un grand projet, tel que le projet de Cotentin Centre (F. Bordet J.F. Parent).

Certaines études ont été faites, mais il s'agit surtout de dresser un inventaire argumenté des actions à entreprendre pour un tel projet, compte tenu de l'expérience acquise. 


\section{Conséquences écologiques \\ de la construction et du fonctionnement de l'usine marémotrice de la Rance \\ (C. Rétière)}

\section{La construction de l'ouvrage}

Hormis les vidanges sanitaires, l'estuaire fut isolé de la mer ouverte durant trois années, de 1963 à 1966. A l'intérieur du bassin, cette période se caractérise par la suppression des marées, des fluctuations importantes de la salinité des eaux, une forte sédimentation accompagnée d'un accroissement du taux de matière organique. Ces nouvelles conditions ont entraîné la disparition quasi totale de la flore et de la faune marine, seules quelques espèces très tolérantes telles les moules ou certains organismes vivant dans les vasières ont prospéré et constitué des populations florissantes.

\section{Le fonctionnement de l'usine}

\section{Incidence sur l'environnement}

Il affecte en premier lieu la "marée " dont le rythme est modifié et l'amplitude réduite. La transformation de l'estuaire à fortes marées en un bassin à faible marnage se traduit par la diminution des surfaces exondables et le recul de la section saumâtre.

Les violents courants de vannage et de turbinage ont légèrement altéré la morphologie de l'estuaire. A proximité du barrage, certains fonds ont été érodés et des bancs de sables déplacés. Dans la partie nord du bassin des sédiments grossiers ont plus ou moins ennoyé les cailloutis qui couvraient le lit de la rivière. Il s'agit dans ce cas de remaniements de dépôts existants et non d'apports nouveaux. Par contre, les longues durées "d'étales" ont favorisé la sédimentation des particules fines d'origine marine dans les anses; le phénomène a surtout été sensible durant les premières années de fonctionnement de l'usine. Toutefois, l'ordre de grandeur des taux de dépôts permet de dire que les processus de sédimentation sont d'importance normale, voire faible, vis-à-vis de ceux que l'on connaît dans certaines baies ou estuaires du littoral.

\section{Conséquences biologiques}

Ce sont les invertébrés benthiques et les populations de poissons qui ont fait jusqu'à présent l'objet des études les plus approfondies. Ces dernières révèlent que sur le plan faunistique le bassin de la Rance est actuellement riche et diversifié.

Après la phase d'isolement de l'estuaire, la recolonisation des fonds s'est réalisée à partir de la mer ouverte à travers le barrage qui a laissé passer les larves pélagiques et les organismes entraînés par les courants.

La recolonisation fut très progressive et dix années ont été nécessaires à l'établissement d'un état d'équilibre, différent bien entendu, de l'état initial. L'occupation des substrats meubles et des roches s'est effectuée en fonction des nouvelles conditions écologiques. La répartition horizontale des espèces entre le barrage et l'écluse du Chatelier est dès lors régie par les nouvelles caractéristiques hydrologiques et leur étagement vertical se réalise par rapport au niveau moyen bas du bassin. Au-dessus de celui-ci se sont installées des espèces observées normalement dans la zone intertidale mais dont l'extension est limitée par le faible marnage; au-dessous, se sont implantées des espèces rencontrées en mer ouverte sur les fonds non exondables. Dans les deux cas, distribution horizontale et étagement vertical il y a eu ajustement biologique aux nouvelles conditions de milieu. Mais la stabilité du nouveau schéma de distribution des organismes est évidemment dépendante de la régularité du rythme de fonctionnement de l'usine marémotrice.

Parmi les espèces peuplant les fonds de roche, l'ormeau, en raison de son intérêt halieutique, fait actúellement l'objet de recherches; la comparaison des résultats relatifs aux populations du littoral et du bassin de la Rance ne laisse pas apparaitre de différences significatives de la densité, des modalités de distribution et de la biologie de cette espèce entre les deux sites.

De plus, le barrage ne semble pas constituer pour les espèces marcheuses de grande taille au comportement migrateur, telles l'araignée de mer, un obstacle infranchissable comme en témoignent les arrivées printanières régulières dans le bassin. D'ailleurs il n'entrave pas non plus le déroulement du cycle biologique de la seiche, migrateur nageur; en effet, même si le franchissement ne se réalise pas sans pertes, la seiche est à la base d'une pêche intensive en Rance à partir du printemps quand les adultes et les immatures qui ont passé l'hiver au large commencent à pénétrer dans le bassin.

La richesse du peuplement piscicole de la Rance est élevée et supérieure, par exemple, à celle de l'estuaire de la Tamar en Angleterre ou de la Baie du Mont SaintMichel. Les populations dominantes regroupent des espèces herbivores, des poissons s'alimentant aux dépens du plancton mais aussi des prédateurs de haut niveau, benthiques, pélagiques ou démersaux. Les prédateurs benthiques sont surtout représentés par des espèces sans intérêt halieutique et les poissons plats, hôtes classiques des estuaires, sont limités à quatre espèces formant des populations clairsemées. La faible représentation de cette catégorie est peut-être due à la difficulté qu'ont ces poissons aux mœurs migratoires ou au moins erratiques à franchir le barrage ou à une compétition alimentaire avec d'autres poissons et (ou) certains crustacés. La question est actuellement à l'étude. Par contre, deux prédateurs pélagiques ont une grande valeur économique : le bar et le lieu jaune. 
A côté des poissons dont la totalité du cycle biologique se déroule en Rance, on peut reconnaitre ceux pour qui le bassin ne joue qu'un rôle de nursery et ceux dont les stades juvéniles et les adultes sont présents sans que les adultes ne s'y reproduisent. En ce qui concerne les deux derniers groupes le recrutement se réalise à travers le barrage. Ces observations vont dans le sens de la perméabilité de l'ouvrage à l'ichtyofaune.

La représentation succinte du sommet de la chaîne trophique en secteur estuarien semble témoigner de relations qualitatives normales qui demandent cependant à être précisées et l'étude de la croissance de certaines espèces (bars, mulets) laisse penser qu'elles trouvent en Rance des conditions favorables de développement.

En conclusion, la faune ichtyologique du bassin apparait variée au niveau spécifique. La zone estuarienne joue son rôle de nursery. Les espèces migratrices (anguilles) et erratiques (bars, mulets, maquereaux...) sont présentes régulièrement. Le franchissement du barrage dans le sens mer-bassin à travers les vannes est évident, le passage dans l'autre sens, par les turbines reste à évaluer.
Enfin, il convient de souligner que malgré la réduction des surfaces exondables, la Rance demeure un site d'acceuil non négligeable pour l'avifaune hivernante dominée par les anatidés et les limicoles.

\section{Conclusions}

Ce bilan écologique sommaire qui traduit une profonde transformation de "l'écosystème Rance " n'est pas pour autant négatif. Toutefois, il faut être conscient que l'équilibre biologique repose sur la notion de régularité du fonctionnement de l'usine. En effet des variations irrégulières des niveaux haut et bas du bassin peuvent avoir des répercussions biologiques drastiques : ainsi en juin dernier elles ont provoqué une chute d'environ $80 \%$ des densités apparentes des populations de jeunes plies...

Tous les enseignements ne sont pas directement transposables à d'autres sites marémoteurs. Il est probable que dans des secteurs où les eaux sont fortement turbides, des dépôts importants modifieront l'environnement benthique.

\section{Organisation des études \\ d'environnement pour le projet \\ Cotentin-Centre \\ (F. Bordet, J.F. Parent)}

L'évaluation de l'impact d'un ouvrage sur le milieu marin impose dans un premier temps une bonne connaissance de ce milieu dans son état naturel. Or, un projet ayant l'emprise de celui du Cotentin-Centre nécessiterait pour ce faire une étude régionale lourde et coûteuse. Nous avons donc, tout d'abord, recensé les impacts possibles afin de faire apparaitre les points sensibles sur lesquels nous ferions porter un effort plus particulier tout en cherchant à définir les grandes caractéristiques régionales et locales des peuplements animaux et végétaux.

Parallèlement, une réflexion a été engagée pour définir les méthodologies les plus appropriées à évaluer les impacts de l'ouvrage et les outils mathématiques devant servir de support à cette démarche.

\section{Recensement des impacts possibles}

La connaissance des impacts sur le milieu vivant passe par la recherche des modifications apportées par l'ouvrage au milieu physique (biotope). Trois grands types de phénomènes nous sont apparus devoir être étudiés:

- modification des champs de courant et de houle dans une zone à préciser autour des ouvrages avec leurs conséquences probables sur la bathymétrie et les caractéristiques sédimentologiques (nature, granulométrie) des fonds et des estrans;

- confinement éventuel de tout ou partie de la masse d'eau dans le bassin en liaison avec le mode d'exploitation et les aléas de fonctionnement. Ce phénomène peut conduire à une évolution de la qualité de l'eau différente de ce qu'elle aurait été en mer ouverte: par exemple échauffement de l'eau favorisé par la faiblesse des fonds, concentration de sels minéraux nutritifs ou de substances polluantes d'origine terrestre dans les bassins, ce risque étant surtout marqué pour l'option englobant la côte :

- isolement des fonds à l'intérieur des bassins avec perturbation de l'équilibre sédimentaire.

Ces modifications du milieu induisent des réponses de la part des peuplements animaux et végétaux. Parmi les possibilités que nous avons envisagés, nous avons retenu celles qui nous paraissent les plus probables ou devoir présenter des inconvénients majeurs.

- développement excessif du phytoplancton dans les bassins, pouvant conduire selon les cas à des phénomènes de type " eaux rouges";

- atteinte aux zones de reproduction côtière de certaines espèces (poissons plats, seiches...) par l'occupation du terrain lui-même, par la modification des fonds recherchés par ces animaux ou par la fermeture des accès à la côte. - modification des peuplements benthiques résultant de l'évolution des sédiments.

Dans ces premières réflexions, nous avons été amenés à inclure également les répercussions que pouvaient avoir les impacts pressentis sur la faune et la flore sur les activités humaines, telles que la pêche et la conchyliculture car toute modification du milieu peut avoir des conséquences sur les espèces exploitées ou sur leur mode d'exploitation. Par exemple, le réhaussement des niveaux de basse mer dans le cas du projet à la côte pouvait 
perturber le travail des conchyliculteurs sur les tables ou les bouchots du bas de l'estran. De même, l'inclusion dans les bassins de fonds activement exploités pour les coquillages, la praire notamment, aurait des répercussions certaines sur la pêche coquillière.

\section{Études destinées à connaître le milieu}

Le Golfe Normano-breton a déjà fait l'objet d'études dans certains domaines tels la courantologie, la sédimentologie ou pour quelques secteurs le peuplement biologique, mais soit par leur secteur géographique, leur échelle ou leur finalité, elles ne correspondaient pas directement à nos besoins tout en constituant pour nous une base de connaissances.

A partir des impacts potentiels que nous avions recensés et des études déjà publiées, nous avons établi un programme de recherche orienté sur les questions que nous aurions à développer dans le cadre de la rédaction d'un dossier d'impact et portant sur les grands domaines suivants : milieu physique, milieu vivant, activités halieutiques.

\section{Le milieu physique}

Les études sur le milieu physique : hydrologie, sédimentologie, morphologie des fonds ont été élaborées en collaboration avec les équipes chargées de la conception des ouvrages car ces paramètres, s'ils sont importants pour l'aspect environnement, sont également indispensables à la définition de la configuration des ouvrages et à leur dimensionnement. Nous ne reviendrons donc pas sur ces études décrites dans la partie consacrée aux aspects techniques, rappelons simplement qu'elles prennent en compte une utilisation ultérieure à des fins de recherche d'impact.

\section{Le milieu vivant}

Pour les études sur le milieu vivant, nous avons retenu deux méthodes d'approche : l'une d'aspect régionale pour dégager les grandes caractéristiques de la faune et de la flore en liaison avec l'hydrologie et la nature des fonds, l'autre centrée sur des problèmes très précis.

Le domaine géographique inventorié lors de cette étude s'étend approximativement de Granville au Havre de St-Germain d'Ay dans la direction Nord-Sud et de la côte au droit des Iles Chausey (incluses) dans la direction Est-Ouest, soit une superficie de l'ordre de $1000 \mathrm{~km}^{2}$ débordant celle du projet proprement dit.

Ces études ont été confiées à des organismes de recherche océanographiques tels le CNEXO, l'ISTPM, ou des laboratoires universitaires.

\section{Domaine pélagique}

Il nous est apparu nécessaire de définir les grandes caractéristiques de la zone au cours du cycle annuel d'en dégager les particularités locales et d'estimer le rôle des apports extérieurs à cette zone : apports côtiers pour les havres et les estrans, apports en provenance du large par les courants de marée, échanges avec le fond du golfe.

Pour cela, six points répartis dans l'ensemble du secteur concerné (figure 1) ont été échantillonnés lors de six campagnes qui se sont déroulées d'avril 1982 à juin 1983. De plus, un point fixe situé en position moyenne (point 24) a été suivi lors de chaque campagne pendant tout un cycle de marée.

Les paramètres inventoriés ont été les suivants :

Hydrologie :

- température,

- salinité,

- sels minéraux nutritifs

$\left(\mathrm{NH}_{4}-\mathrm{NO}_{2}-\mathrm{NO}_{3}-\mathrm{PO}_{4}-\mathrm{Si}\right)$,

- matières en suspension avec une attention particulière portée à la tranche d'eau située à proximité du fond, siège des mouvements sédimentaires.

Phytoplancton

- évaluation.de la biomasse par mesure des pigments chlorophyliens,

- détermination et dénombrement des principales espèces,

- évaluation de la production primaire potentielle par la méthode au carbone 14 .

\section{Zooplancton}

- évaluation globale de la biomasse zooplanctonique.

\section{Domaine benthique}

Comme pour le domaine pélagique, nous avons cherché à dégager les grandes caractéristiques du secteur en dressant une cartographie à grande échelle des peuplements benthiques en s'attachant particulièrement aux peuplements les plus productifs tels que les ceintures algales, les herbiers, les sables fins à annélides et les sables grossiers à bivalves.

Cette cartographie a été réalisée à partir de techniques donnant une vue globale de la répartition des différentes communautés, tels que télévision sous-marine et sonar latéral pour les fonds immergés et photographie aérienne en couleur ou infrarouge pour les estrans. Des prélèvements sont réalisés simultanément sur le terrain en des points représentatifs pour caler et étalonner les enregistrements. Ceci permet de donner une estimation réaliste de la biomasse des différents peuplements.

En plus de cette cartographie générale, nous avons porté un effort spécifique sur trois points sensibles du secteur cotentin :

- le développement d'une algue de la famille des sargasses dont la prolifération peut être une gêne pour l'exploitation;

- les exigences écologiques des bivalves, ressource halieutique essentielle de la zone (praire, palourde, amande) notamment en ce qui concerne la qualité du substrat sédimentaire;

- répartition du maerl, sédiment calcaire grossier d'origine algale et résistance à l'envasement des espèces qui le constituent.

\section{Activités halieutiques}

Nous avons considéré cette partie sous deux aspects : la ressource et l'activité de pêche et de conchyliculture. 


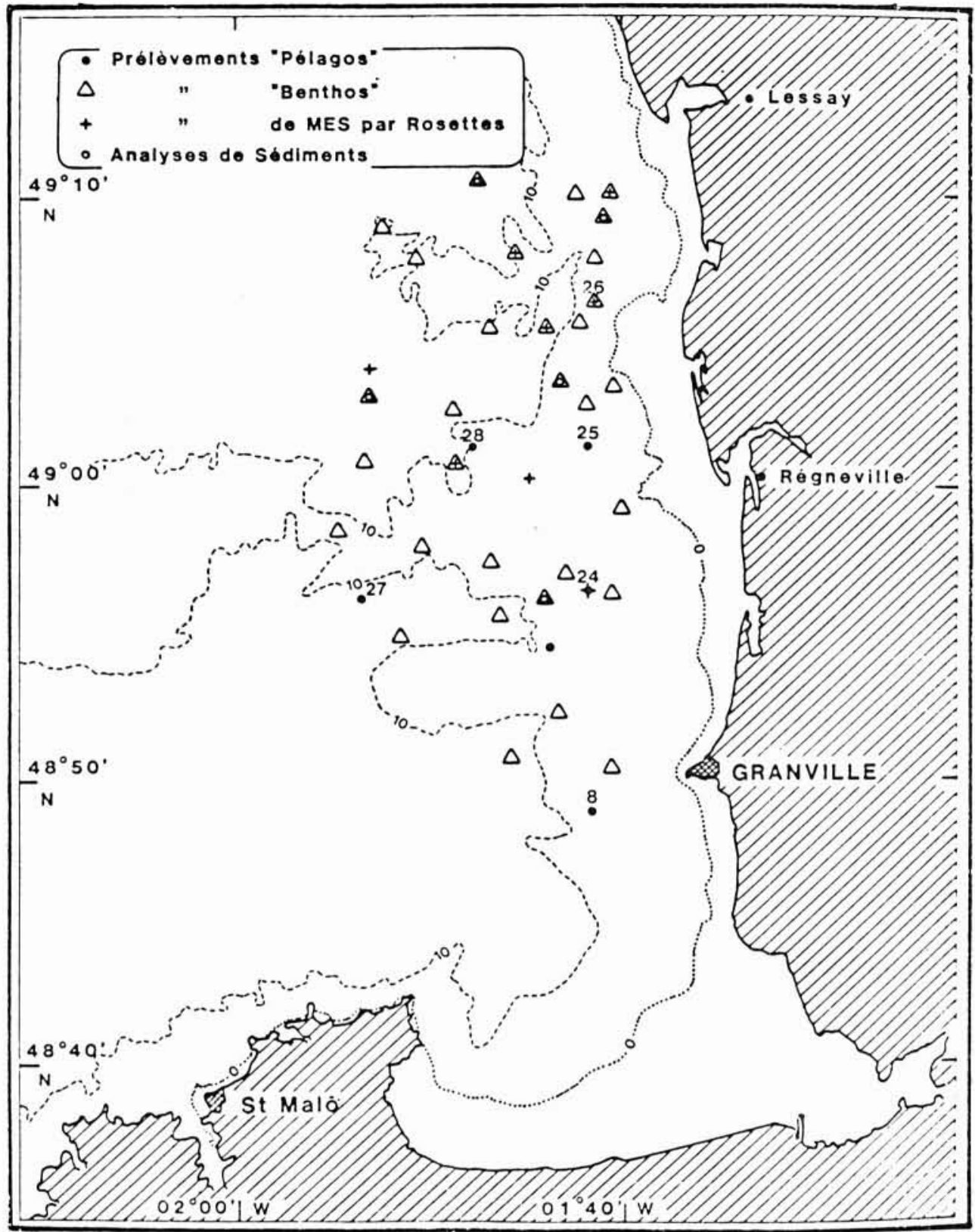

Cotentin Centre Positionnement des points étudiés.

\section{Ressources halieutiques}

Nous avons cherché à situer les ressources halieutiques, à en dresser un inventaire avec, lorsque cela était possible, une quantification des abondances. Une partie de cette étude a été consacrée à la localisation des zones de frayère (poissons plats, seiche...) et de nourricerie des juvéniles.

Parmi les espèces recensées, deux ont fait l'objet d'investigations plus approfondies en raison de leur grande importance économique régionale. Ce sont la praire et le buccin pour lesquelles nous avons cherché à connaitre la localisation précise des gisements, l'état du stock et sa dynamique.

\section{Activités de pêche et de conchyliculture}

Pour la partie "pêche ", nous avons cherché à connaître au moyen d'enquête auprès des professionnels et par survol aérien la localisation des zones de pêche, l'intensité de l'activité sur les différents secteurs, les quantités débarquées selon les espèces, et les données socio-économiques classiques sur l'activité du Quartier Maritime de Granville.

Pour la partie conchyliculture, particulièrement développée sur cette portion de littoral, nous avons utilisé la même approche, c'est-à-dire enquêtes auprès des professions et survols aériens dans le but d'estimer la production globale du secteur ainsi que les modes d'exploitation pratiqués par les conchyliculteurs.

\section{Évaluation des impacts de l'ouvrage}

Ces études qui sont de la responsabilité du maître d'ouvrage sont réalisées par Électricité de France - Direction 
de l'Équipement (Région d'Équipement Clamart, et le Département Sites-Environnement-Information) et Direction des Études et Recherches (Laboratoire National d'Hydraulique et Département Environnement Aquatique et Atmosphérique).

Leur objectif est de prévoir, à partir de la situation naturelle de la configuration des ouvrages et du mode d'exploitation de l'usine, les modifications induites dans le milieu marin.

La première phase de cette démarche consiste à rechercher la nouvelle configuration des champs de courant qui conditionne les impacts sur les différents compartiments du milieu marin.

Impact sur le milieu physique : Les modifications apportées au champ des courants seront déterminées au moyen de modèles numériques à différentes échelles afin de décrire la nouvelle configuration tant à l'extérieur des bassins qu'à l'intérieur. Les résultats de cette première étude seront directement utilisés pour rechercher à partir des lois de charriage et de la circulation locale des masses d'eau, les nouveaux équilibres sédimentaires qui résulteront de l'implantation et du fonctionnement des ouvrages : répartition des sédiments de fond, évolution de la côte.

Impact sur le milieu vivant : L'impact sur le milieu vivant est la conséquence des modifications apportées au milieu physique. Cette partie de l'étude d'impact vient donc en aval de l'étude du milieu physique dont elle utilisera les résultats. L'évaluation quantitative de l'impact d'un ouvrage sur le milieu vivant est encore aujourd'hui un exercice difficile car la dynamique des différents peuplements et les échanges entre les multiples composants d'un écosystème sont mal connus, a fortiori dans une zone aussi complexe que l'est la zone côtière. C'est pour cela que la finesse de l'étude ainsi que les méthodes d'investigation seront différentes selon le cas traité.

Dans le domaine pélagique, à l'intérieur des bassins, le problème principal est celui de l'évolution des paramètres de la qualité de l'eau, en particulier la température et les sels minéraux nutritifs. Cette étude sera menée au moyen de modèle mathématique dérivé de ceux utilisés pour les retenues de barrage. A partir de ces résultats, le risque d'eutrophisation ou d'apparition d'eaux rouges sera estimé de façon empirique à partir de l'expérience que l'on a sur les mécanismes de formation de tels phénomènes. A l'extérieur des bassins, il nous faudra évaluer les modifications des apports nutritifs (indispensable à la nutrition des animaux benthiques) car les masses d'eau "océaniques" venant du large et "côtières " venant du fond du golfe n'ont pas la même potentialité nutritive et les ouvrages modifieront la circulation de ces courants. Cette question sera abordée de façon globale en faisant un bilan des apports à partir des données du modèle de courants.
Dans le domaine benthique, à l'intérieur comme à l'extérieur des bassins, l'évolution des peuplements benthiques est liée à l'évolution de la nature des sédiments. A partir des résultats de l'étude sédimentaire, en tenant compte des exigences des principales espèces composant les peuplements benthiques en matière de qualité de leur substrat, nous établirons une nouvelle répartition de ces peuplements. Une approche plus quantitative pourra être éventuellement entreprise en considérant la biomasse et la productivité moyenne des différents peuplements pour évaluer globalement un gain ou un déficit de matière vivante sur le secteur perturbé.

Une démarche analogue sera conduite pour les estrans.

Impact sur les activités halieutiques: L'impact sur les activités halieutiques constitue un échelon de complexité supérieur par rapport à l'impact sur le milieu vivant car les ressources halieutiques sont généralement constituées d'animaux occupant les niveaux supérieurs des réseaux trophiques donc intégrant les perturbations à tous les niveaux inférieurs et sur lesquels intervient la diversité des activités humaines (pratiques de pêche, de récolte, de culture...) avec leurs adaptations au milieu, leurs traditions, leurs poids et leurs difficultés économiques. Ce domaine est donc celui où une prévision quantitative des impacts, si elle apparaît le plus nécessaire, est également le plus difficile à établir. Nous tenterons cependant cette approche en essayant d'évaluer les atteintes portées aux ressources, principalement les coquillages et les perturbations apportées aux modes d'exploitation telles que la difficulté ou l'impossibilité à travailler sur les lieux de pêche traditionnels, la réduction des surfaces disponibles pour les conchyliculteurs ou la modification des pratiques d'élevage.

Utilisation des bassins à des fins non énergétiques: Dans le bilan global des impacts des ouvrages, on peut intégrer des utilisations possibles des ouvrages à d'autres fins que la production d'énergie. En effet, ils constituent un vaste plan d'eau protégé de la houle, propice à un certain nombre d'activité, car accessibles par la terre comme par la mer. Parmi celles-ci, trois retiennent particulièrement l'attention :

- les activités nautiques, telle la planche à voile, dont on peut constater le développement dans le bassin de la Rance;

- la pêche : la superficie des bassins et la richesse des fonds permet de penser que la pêche pourra continuer à s'y exercer dans des conditions plus favorables;

- les cultures marines : le milieu protégé que constituent les bassins sera éminemment favorable au développement de cultures marines : huitres et moules en eau profonde, poissons en cages flottantes. 


\section{Discussion}

Président : M.R. VULSER

Le deuxième rapport a comporté trois interventions. La parole a été d'abord donnée à M. MAssonNET, Chef de la Division Environnement de la Direction de l'Equipement d'E.D.F., qui introduit le sujet. M. RÉTIĖRE, Directeur du Laboratoire Maritime de Dinard, dépendant du Muséum d'Histoire Naturelle a décrit les conséquences écologiques de la construction, puis de l'exploitation de l'usine de La Rance. Enfin, M. BORDET, appartenant à la même Division que M. MASSONNET, a énuméré les études lancées pour évaluer l'impact du projet Cotentin Centre.

M. le Président remercie les trois rapporteurs et ouvre la discussion après avoir remarqué que, sous réserve d'aborder avec soin et respect les problèmes d'influence sur l'environnement, ceux-ci peuvent être bien maitrisés.

M. RECEVEUR: Vous avez indiqué que l'installation de la Rance n'était pas étanche aux poissons. Sait-on s'ils passent uniquement par les vannes ou aussi par les groupes? Quel est alors le taux de mortalité ?.

M. RÉTIERE Pour l'instant on sait simplement que les poissons passent sans doute avec quelques pertes. On étudie en ce moment par oủ ? et comment? M. Bosc cite des essais américains qui prouvent que le fonctionnement des groupes en orifice n'a aucune influence sur le passage des poissons.

M. KÉRISEL: On ne peut que se féliciter des conclusions, dans l'ensemble optimistes, de l'exposé de M. Rétrére lorsqu'on le compare avec celui du barrage d'Arzal (sur la Villaine), dont certaines conséquences sur l'environnement ont été majeures.

M. POIREL rappelle un projet qui utilisait la différence de phase et d'amplitude de la marée entre les côtes Est et Ouest du Cotentin. M. BANAL indique que ce projet conduisait à une production très faible pour un investissement et des problèmes d'environnement considérables.

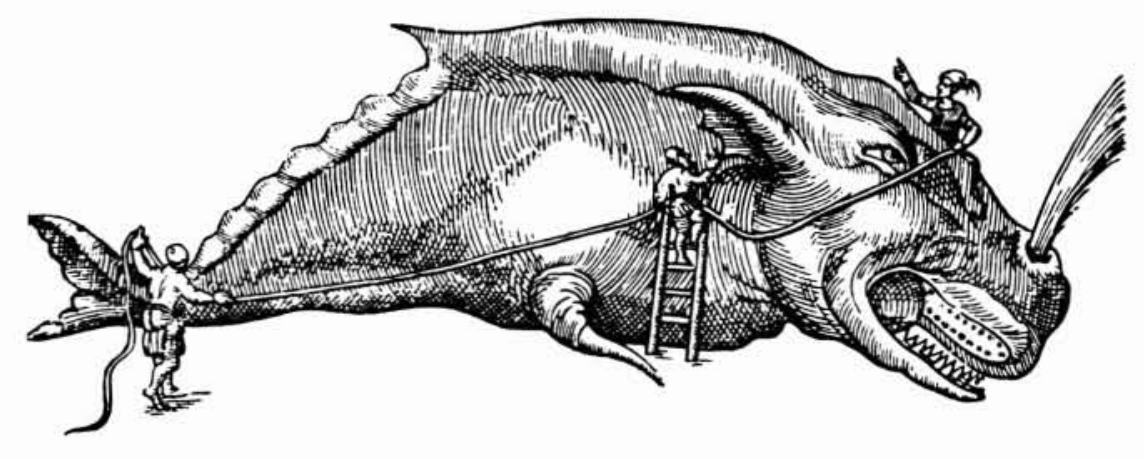




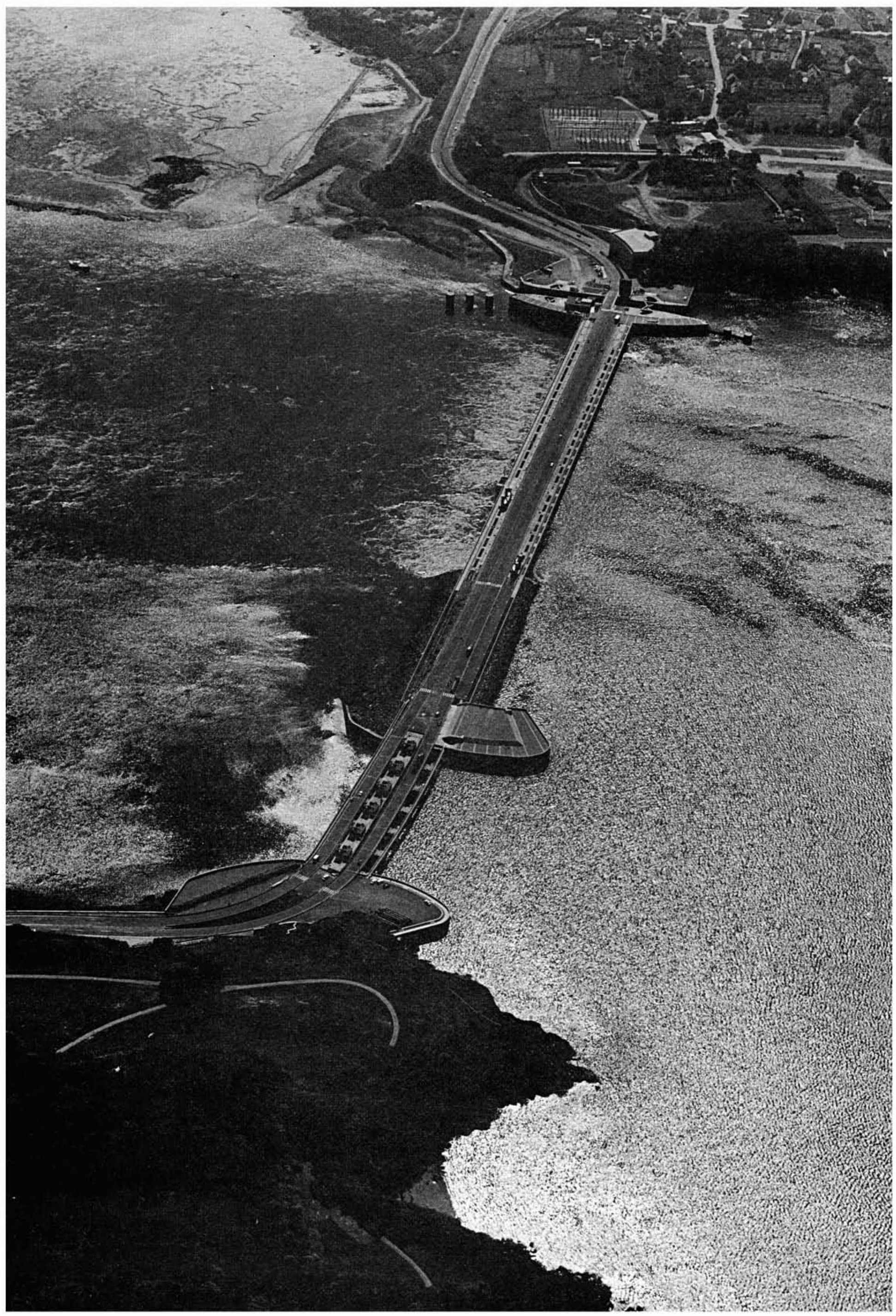

